\title{
Leaf growth dynamics of two congener gymnosperm tree species reflect the heterogeneity of light intensities given in their natural ecological niche
}

\author{
I-LING LAI ${ }^{1}$, HANNO SCHARR $^{2}$, ANDRES CHAVARRIA-KRAUSER ${ }^{2}$, RALF KÜSTERS $^{2}$, JIUNN-TZONG WU $^{1,3}$, \\ CHANG-HUNG CHOU ${ }^{4}$, ULRICH SCHURR ${ }^{2} \&$ ACHIM WALTER ${ }^{2}$ \\ ${ }^{1}$ Institute of Ecology and Evolutionary Biology, National Taiwan University, Taipei 106, Taiwan, ${ }^{2}$ Institute of Chemistry and \\ Dynamics of the Geosphere ICG-III: Phytosphere, Research Center Juelich GmbH, 52425 Juelich, Germany, ${ }^{3}$ Research Center \\ for Biodiversity and Institute of Botany, Academia Sinica, Taipei 115, Taiwan and ${ }^{4}$ Department of Tropical Agriculture \& \\ International Cooperation, National Pingtung University of Science and Technology, Pingtung 912 Taiwan
}

\begin{abstract}
Chamaecyparis obtusa var. formosana and Chamaecyparis formosensis are congener gymnosperm tree species native to Taiwan cloud forests; occupying different niches there. While the seedlings of $\boldsymbol{C}$. formosensis occur predominantly under bright conditions in large forest gaps, seedlings of $C$. obtusa var. formosana are mainly found below the canopy of mature forests or in small gaps. It is well known that congener species occupying different niches typically differ in several ecophysiological and morphological traits, but the differences in growth dynamics of such species are still totally unclear, as the diurnal growth dynamics of gymnosperm leaves have not been investigated before. Modern methods of digital image sequence processing were used in this study to analyse the leaf growth dynamics of the two species. We found that both species show similar base-tip gradients and pronounced diurnal growth rhythms with maxima in the evening. Differences between the two species concerning their growth dynamics correlated closely with their ecological amplitudes and abundances. Chamaecyparis obtusa var. formosana grew faster than $C$. formosensis in low light intensity, typical for closed-canopy situations, and reacted quickly by increasing or decreasing growth rate when light intensity changed within a range typically found below small canopy gaps. In contrast to this, $\boldsymbol{C}$. formosensis grew better in light intensities typical for open vegetation situations, but reacted slower towards changes of light intensity. Based on those results, the hypothesis can be developed that fluctuations of leaf growth dynamics reflect heterogeneities of the light environment within the niche occupied by a given species.
\end{abstract}

Key-words: Chamaecyparis; congener gymnosperm; image processing; light; niche; relative growth rate.

Correspondence: Achim Walter. Fax: 49 2461612492; e-mail: a.walter@fz-juelich.de

1496

\section{INTRODUCTION}

Plants are capable of tolerating a wide range of light environments by acclimation or adaptation of their photosynthetic apparatus. Acclimation of leaves growing in sun or shade conditions has been studied extensively (e.g. Öquist et al. 1992; Anderson, Park \& Chow 1997; Barth, Krause \& Winter 2001; Walters 2005). Sun and shade leaves differ in a wide range of traits, such as xanthophyll cycle pigments (Demmig-Adams \& Adams 1992; Demmig-Adams 1998; Niinemets et al. 1998), Chlorophyll $a / b$-ratio, light-harvesting complex (LHCP) I/II ratio (Bailey et al. 2001) and leaf anatomy (Oguchi, Hikosaka \& Hirose 2003). Those traits enable plants to deal with excessive irradiation or to increase light efficiency under low light conditions. Not all species are uniformly adapted to tolerate high (or low) light conditions, resulting in shade-intolerant and shade-tolerant species (Lei \& Lechowicz 1990,1998). They can better cope with constant levels of high or low light intensity to occupy different niches as pioneer or late-succession species (Barker \& Brown 1997; Bassow \& Bazzaz 1997; Davies 1998).

Investigations of growth reactions of species are often restricted to observations of the occurrence of a given species in a given natural light climate or to measurements of classical growth parameters such as leaf area ratio or relative growth rate measured at intervals of several days in shading experiments (Dalling, Lovelock \& Hubbell 1999). Dynamic growth parameters - such as principal features of the diurnal course or immediate responses towards changes of light intensity - have not yet been investigated in most ecological studies. Furthermore, the growth dynamics of gymnosperm leaves have not been investigated before due to their typically small size and slow growth rate. It is unknown whether they show diurnally oscillating growth intensity, in a similar way to angiosperm leaves (Walter \& Schurr 2005). Novel measurement techniques (Schmundt et al. 1998; Walter, Feil \& Schurr 2002a; Walter et al. 2002b; van der Weele et al. 2003) now allow us to link growth traits with physiological parameters, both analysed in temporal 
ranges of minutes to hours and spatial scales of suborgan levels (Walter \& Schurr 2005). Yet, it is still an open question, whether fluctuations of light intensity are correlated with fluctuations of plant growth, and if so, whether plants have evolved growth traits adapting them to a given niche.

For this study, we selected two species from the gymnosperm genus Chamaecyparis that are adapted to different ecological niches in montane areas of Taiwan, where fog frequently occurs (Su 1984). Chamaecyparis obtusa var.formosana and C. formosensis (Lee 1962; Liu 1975) are dominant species in these forests. They are distributed in overlapping areas and have similar life history and life form. Yet, plants of C. obtusa var. formosana mostly regenerate from the seedling bank of the forest floor or in small canopy gaps, whereas plants of $C$. formosensis regenerate in large gaps and disturbed open areas, showing a more pioneer-species-like behaviour (Chang 1963; Liu 1975; Lin \& Lai 1999). This difference was suggested to correlate with the intensity of irradiation received by seedlings (Chen 1968; Ho 1988) and it was hypothesized that the two species have diverged to partialize the occupied niches in order to eliminate heavy intrageneric competition.

The first aim of this study was hence to meet the experimental challenge of analysing the diurnal pattern of gymnosperm leaf growth variation. The second aim was to compare the leaf growth dynamics of both species in different light regimes to get an indication about physiological differences that might lead to the different ecological amplitudes of the two species.

\section{MATERIALS AND METHODS}

\section{Growth conditions}

Seeds of C. obtusa var. formosana and C. formosensis were collected from an area in the cloud forest of north-east Taiwan. The seeds were sown in a nursery and the seedlings were well supplied with nutrients and water. The 3-year-old seedlings, about $25-40 \mathrm{~cm}$ high, were transplanted into 1.5 L pots. Thereafter, they were grown for several months in growth cabinets with a relative humidity of $60-70 \%$ and temperatures between 20 and $25^{\circ} \mathrm{C}$ under extreme low, low, medium, high, and extreme high light regimes: 10, 30, 75-100, 150-300 and $400 \mu \mathrm{mol} \mathrm{m}^{-2} \mathrm{~s}^{-1}$ photosynthetic photon flux density (PPFD) with 12/12 h day/night period. These light regimes mimicked the specific conditions at the natural site, where light intensity is generally very low due to the prevailing clouds and accompanying fog. The PPFD of the extreme low light regime corresponded to the average light intensity on a forest floor covered by broad-leaved trees. The PPFD of the low light regime corresponded to the average diurnal light intensity in the understorey of a Chamaecyparis forest. The PPFD of the medium light regime corresponded to the average situation close to small gaps caused by tree fall or by the construction of logging roads. The high light regime mimicked the PPFD of a large disturbed area in the montane cloud zone in Taiwan, such as regeneration sites of landslides or forest fires. The extremely high light regime was comparable with the average diurnal value under totally clear conditions outside the cloud zone in the field (Lai, unpublished results). After 2 months, most of the plants under extreme low and extreme high light regimes looked unhealthy, thus we only used the healthy individuals that were grown under low, medium and high light regimes for this study. During the experiments of our study, all leaves that had partly developed before transplanting were fully emerged and all growing leaves were formed in the described light conditions.

\section{Gas exchange measurements}

The gas exchange rates of small seedling branches with totally $3-5 \mathrm{~cm}^{2}$ mature and healthy foliage were measured with a portable computerized open-system infrared gas analyser (Li-6400; Li-Cor, Inc., Lincoln, NE, USA). A cold light source (6400-02 red-blue LED) was mounted on the leaf chamber as the source of variable light. All measurements were made in the growth cabinet where the seedlings were raised with cuvette $\mathrm{CO}_{2}$ concentration set at $350 \mathrm{ppm}$ and relative humidity above $60 \%$. The foliage had been dark-incubated in the leaf chamber for at least $20 \mathrm{~min}$ before recording the data. $\mathrm{CO}_{2}$ assimilation rates were recorded at PPFD values of $0,4,8,12,16$ and $20 \mu \mathrm{mol}$ $\mathrm{m}^{-2} \mathrm{~s}^{-1}$. Then PPFD was switched to $100 \mu \mathrm{mol} \mathrm{m} \mathrm{m}^{-2}$. Measurement time was at least $20 \mathrm{~min}$ before light intensity was increased in order to assess the highest potential assimilation rate in each light condition. Gas exchange was recorded at PPFDs of 100, 200, 400, 600, 800, 1000, 1200, 1600, $2000 \mu \mathrm{mol} \mathrm{m}^{-2} \mathrm{~s}^{-1}$. Photosynthetic light response curves were produced from the gas exchange data of each seedling. At least three seedlings from each species were measured in each light regime. Apparent quantum yield $\left(Q_{\mathrm{y}}\right)$, dark respiration rate $\left(R_{\mathrm{d}}\right)$, light compensation point (LCP), and maximum $\mathrm{CO}_{2}$ assimilation rate $\left(A_{\max }\right)$ were calculated from the photosynthetic light response curves.

\section{Image acquisition and processing}

Leaves of the second or third branch from the shoot apex of the seedlings were selected for the imaging growth measurements. Those leaves had emerged and developed in the chosen light regime. Apices of growing branches were mounted gently between two microscope slide glasses (Fig. 1a, b \& c). Two near-infrared LED-arrays (wavelength $940 \mathrm{~nm}$ ) were positioned next to the branch to ensure constant image brightness throughout night and day. Nearinfrared images of the foliage at the branch apex were acquired every 5 min by a CCD camera (Sony XC-75; DBS; Bremen, Germany) from underneath the leaf. The distance between camera and foliage was about $6.5 \mathrm{~mm}$ (Fig. 1c);

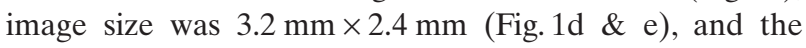
CCD-resolution was $640 \times 480$ pixels. Using this magnification, the best results were obtained when in the first image of an image sequence, two to four layers of opposite leaves were visible. This was on the one hand enough foliage to allow reliable calculations of growth rates from velocities 

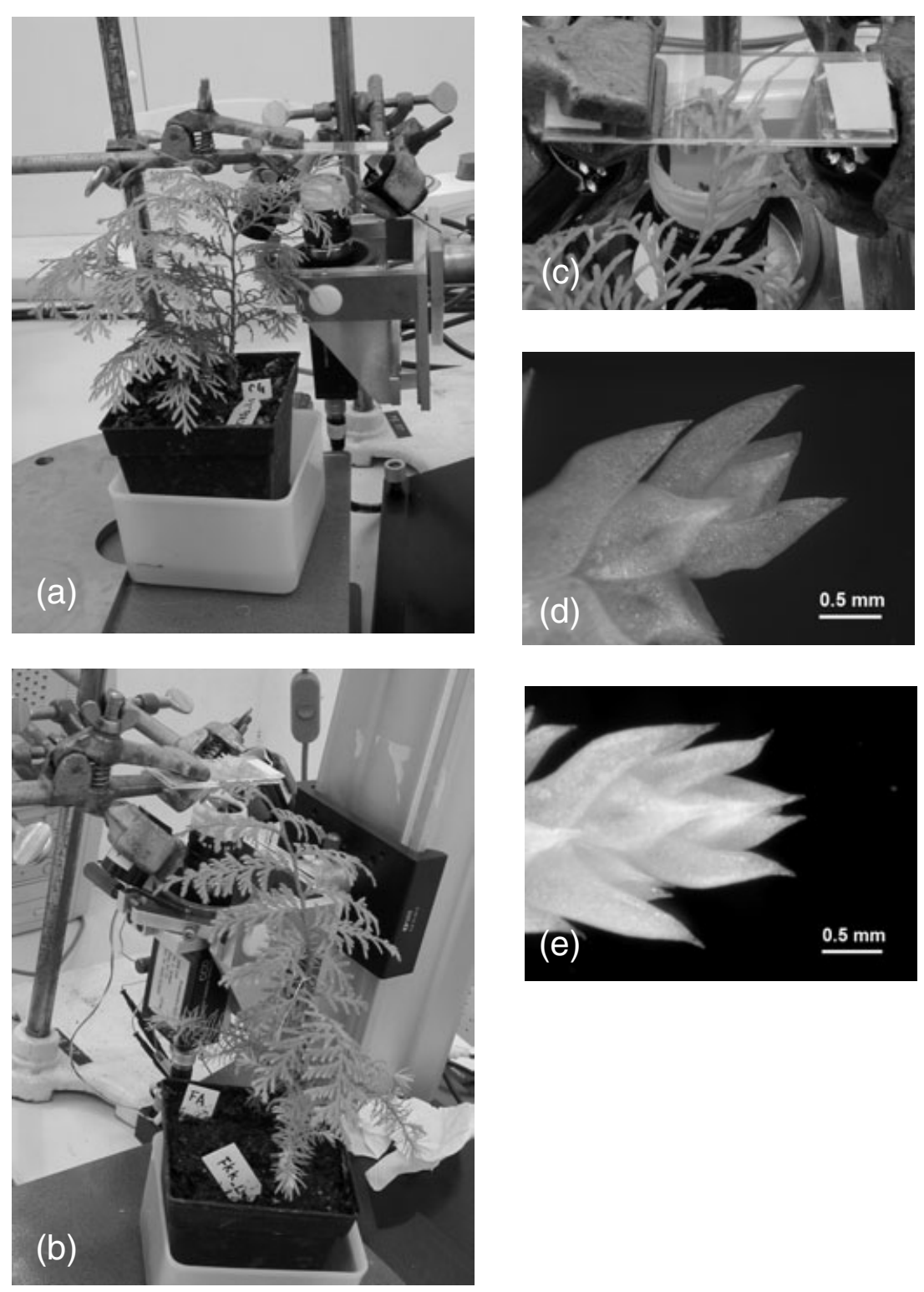

Figure 1. Plant phenology and image acquisition process. The image acquisitions were performed with seedlings of Chamaecyparis obtusa var. formosana (a) and C. formosensis (b). The tip of a seedling branch was fixed between two slide glasses (c). Typical images used for analysis of C. obtusa var. formosana (d) and C. formosensis (e). at different points within the foliage (see below) and on the other hand, there was still enough space between foliage apex and image borderline (at least one-third of the image area) for the foliage to grow within the chosen image frame. Typically, after $3 \mathrm{~d}$, the foliage reached the border of the image. To acquire longer sequences, the camera was then shifted.

Within the black/white image sequence, natural grey value structures of the foliage are shifted with time because of the movement and growth of the leaves. To calculate distributions of relative growth rates (RGR) within the foliage, displacement vector fields for each image have to be calculated first. Any structure with a suitable grey value contrast to its neighbourhood results in oriented grey value structures in image stacks. The slope and orientation of those trajectories in the spatio-temporal neighbourhood of a central pixel were analysed via an optical flow approach (structure-tensor algorithm) and lead to a velocity vector for the motion of the central pixel. After determination of velocities for all suitable pixels in each image and within the entire sequence, missing information is filled in by interpolation for foliage regions showing too little grey value structure. Areal relative growth rates are now calculated at each pixel by taking the divergence of the velocities of neighbouring pixels (for detailed information see Schmundt et al. 1998; Walter et al. 2002a, b). Results can be averaged spatially and temporally on demand and are displayed as colourcoded maps or as time-series of selected areas of interest (AOI; see Figs 3b, d \& 4a). Sub-algorithms allow the choice of an AOI in the beginning of the sequence and to extract time-series for relative growth rates for the foliage part of the initial AOI as borderlines are growing along with the moving and expanding organ (Fig. 3; Scharr 2004). For most purposes of this study, 1-h average values of RGR were calculated. For representation of 'normalized' growth data, 1-h average values of RGR were normalized with respect 
to the average 24 -h value of RGR (average $\mathrm{RGR}=100 \%$ ). For statistical analyses, the results of at least three plants per species and per light treatment were used.

\section{Transfer of plants between low and medium light regimes}

After growth conditions had been established and the basic features of the diurnal growth pattern had been investigated, we recorded the change of RGR time-series, when the seedlings were transferred from the light condition to which they were acclimated to a different one. Two seedlings of each species which had been grown for at least 8 weeks in low and medium light regimes, respectively, were transferred to the opposite light regime (low to medium and medium to low). RGR was monitored for $6 \mathrm{~d}$ before and after the transfer. The hourly RGR of the two replicates was averaged to represent the response of the species to a sudden change in light regime.

\section{Statistical analysis}

All parameters measured in the gas exchange experiment and hourly averaged RGR time series were examined with two-way analysis of variance (ANOVA) with 'species' and 'light regime' as fixed factors (STATISTICA version 6.0, StatSoft, Inc., Tulsa, OK, USA). The difference of means was compared using Fisher's Least Significant Difference (LSD) test.

\section{RESULTS}

\section{Basic characteristics of diurnal leaf growth pattern}

To establish optimal laboratory growth conditions for the seedlings, some characteristic parameters of their photo- synthesis were used in addition to the observations concerning their overall growth performance at different light regimes. Although LCP increased with light intensity in both species, highest $R_{\mathrm{d}}, A_{\max }$ and $Q_{\mathrm{y}}$ were found in both species when they were grown in the medium light regime (Fig. 2). Gas exchange of both species was inhibited in the 'high' light condition applied in this study. There were significant differences for each parameter between light treatments $(P<0.05)$, whereas interspecific comparisons showed relatively similar results with the exception of $A_{\max }$ in the medium light regime: There, $C$. formosensis had a markedly higher $A_{\max }$, indicating that this species acclimates much better to changes in light intensity.

In optimal growth conditions (medium light regime), strong and highly repetitive diurnal RGR patterns were found for leaves of both species (Fig. 3). In both species, clear spikes of negative expansion were found daily. There was no immediate difference between the growth dynamics of both species. Analysis of spatial leaf growth distribution within single leaves and within the foliage region used for most figures of this paper showed a high degree of patchiness, but again no clear difference was found between both species (Fig. 4). Both species showed the highest RGR at the day-night transition, and had a relatively stable RGR during the last $6 \mathrm{~h}$ of the night (Figs 3, 4b \& c). Clear basetip gradients were present throughout the entire diurnal course with highest growth rates found at the leaf base (Fig. 4b \& d). When negative expansion occurred transiently, the base-tip gradient changed its sign and highest negative expansion rates occurred at the leaf base (Fig. 4b).

\section{Diurnal leaf growth patterns in different light regimes}

The comparison between relative leaf growth rates in three different light intensities showed for both species, that
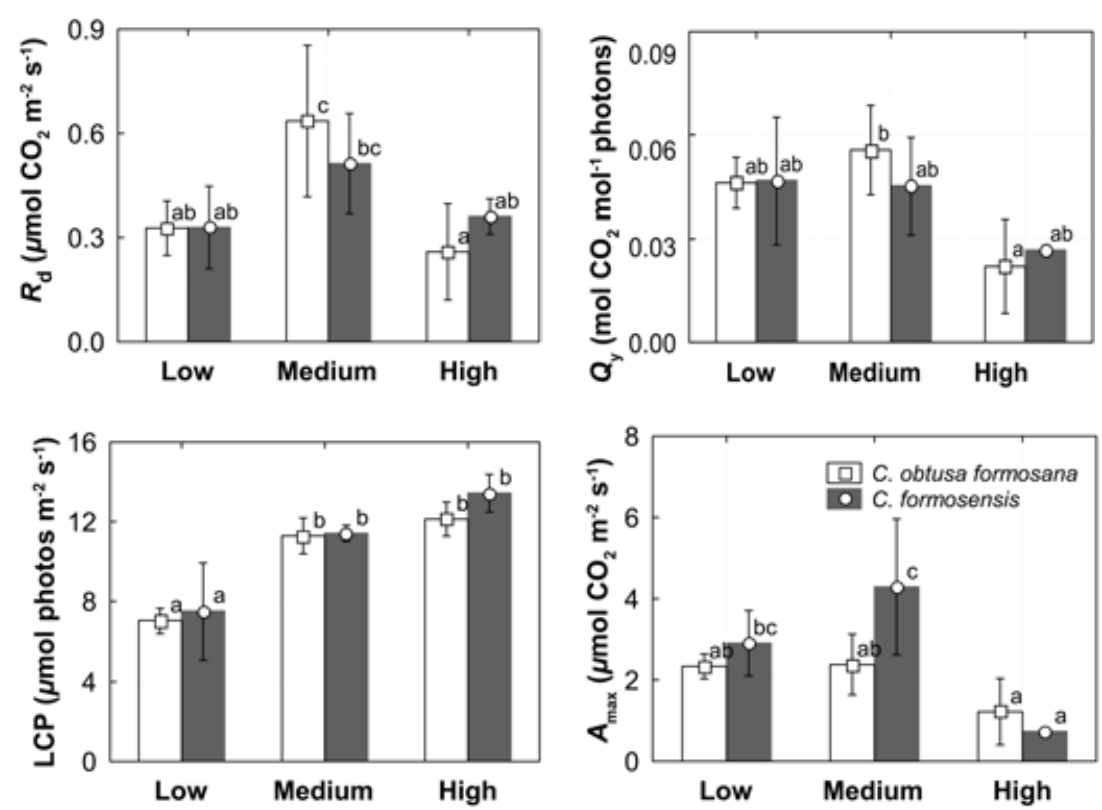

Figure 2. Comparison of photosynthetic characteristics between Chamaecyparis obtusa var. formosana and $C$. formosensis seedlings under different light regimes $(n \geq 3$; standard deviation and results from Fisher's LSD-test are shown). Dark respiration rate $\left(R_{\mathrm{d}}\right)$, light compensation point (LCP), apparent quantum yield $\left(Q_{\mathrm{y}}\right)$, and maximum $\mathrm{CO}_{2}$ assimilation rate $\left(A_{\max }\right)$ are shown. 


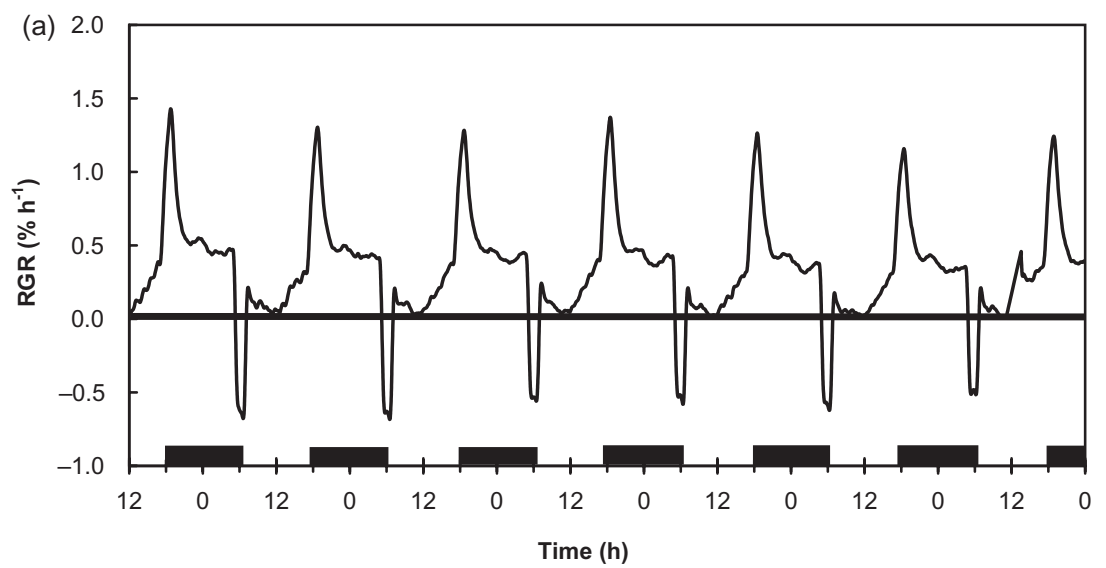

(b)
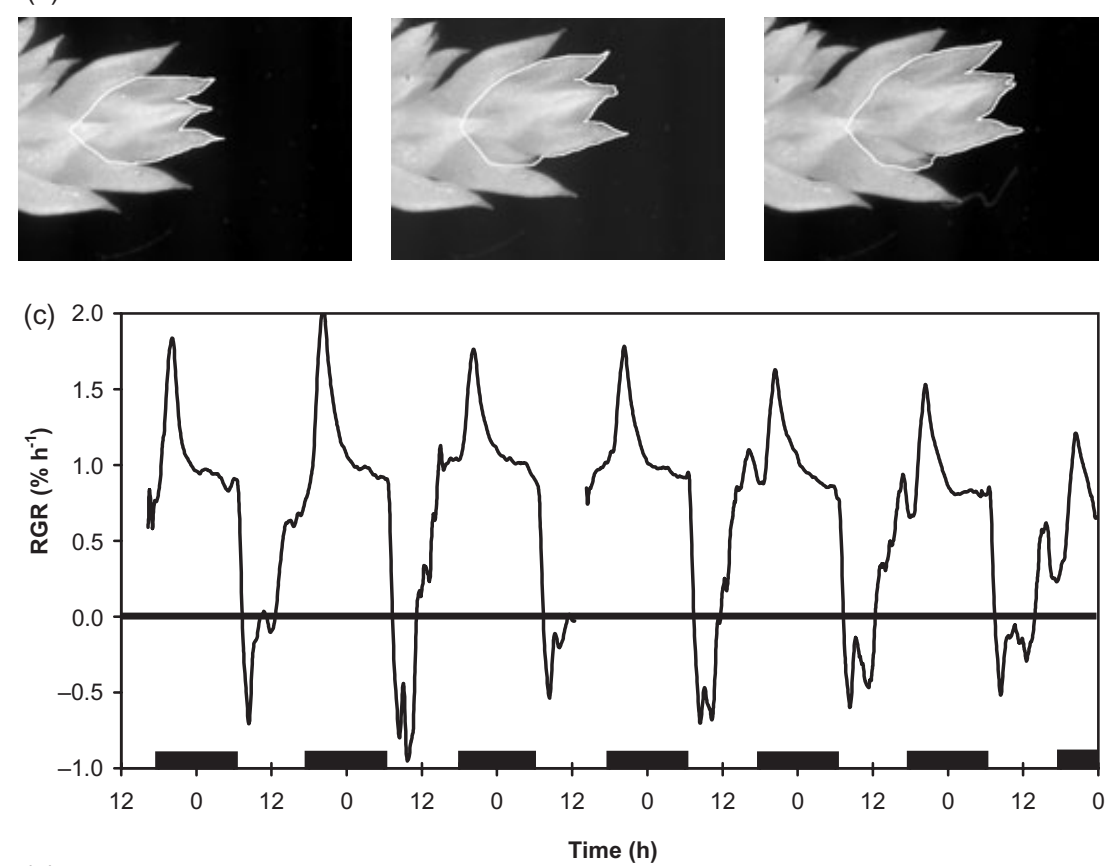

(1)
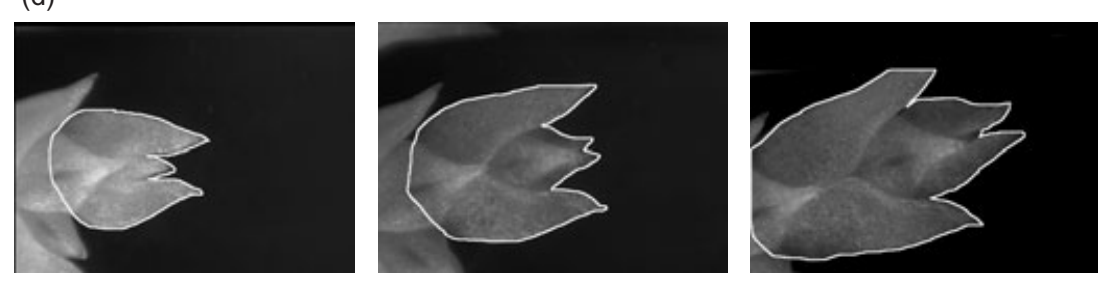

Figure 3. Relative growth rate (RGR) of young foliage. (a) RGR of Chamaecyparis formosensis throughout $7 \mathrm{~d}$ under medium light regime. (b) Analysed area of interest from (a) at days $1,3.5$ and 7. (c) RGR of $C$. obtusa var. formosana throughout $7 \mathrm{~d}$ under medium light regime. (d) Analysed area of interest from (c) at day 1,3.5, and 7. growth was strongly retarded in high and low light conditions (Fig. 5). Optimal growth rate was found under medium light conditions. This corresponded to the results of gas exchange analysis. Growth rate increased almost monotonically throughout the day and decreased almost monotonically throughout the night. Negative expansion occurred in both species at the night/day transition for every light regime and was most obvious in the medium light regime (Fig. 5). Chamaecyparis obtusa var. formosana reached higher RGR-values than $C$. formosensis in the low light regime (peak values of 0.4 versus $0.2 \% \mathrm{~h}^{-1}$ ) whereas C. formosensis reached higher RGR values than C. obtusa var. formosana for medium and high light conditions (peak values of 2.0 versus $1.5 \% \mathrm{~h}^{-1}$ and of 0.6 versus $0.3 \% \mathrm{~h}^{-1}$ in medium and high light intensity, respectively).

To compare the growth dynamics of both species at different light regimes, 1-h average RGR values were normalized (Fig. 6). Both species had an almost identical diurnal growth rate cycle in low light conditions. In medium and high light conditions, C. obtusa var. formosana showed a much more 'disturbed' growth rate cycle than $C$. formosensis with values extending into the negative region for a longer time and with greater relative intensity. In high light conditions, leaves of C. obtusa var. formosana shrank 
(a)
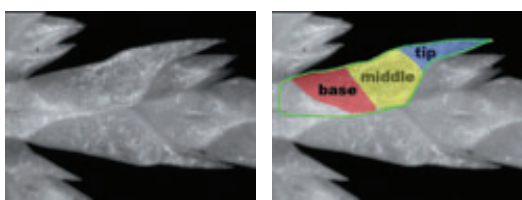

(b)

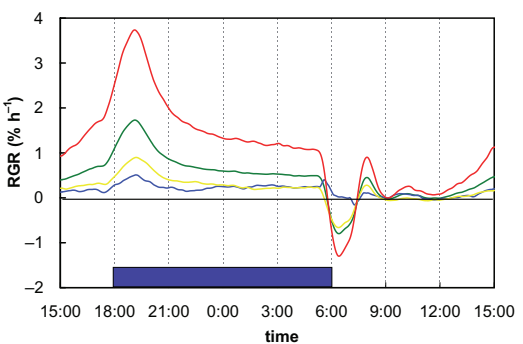

(c)
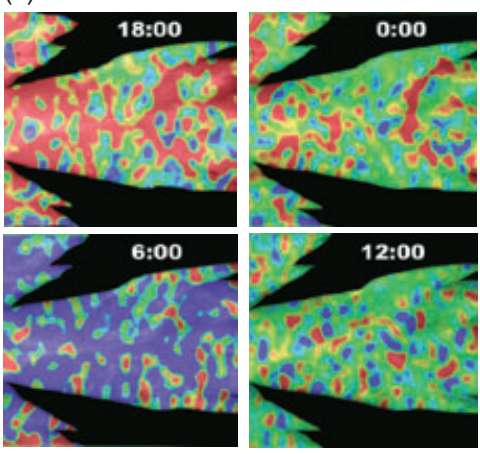

(d)

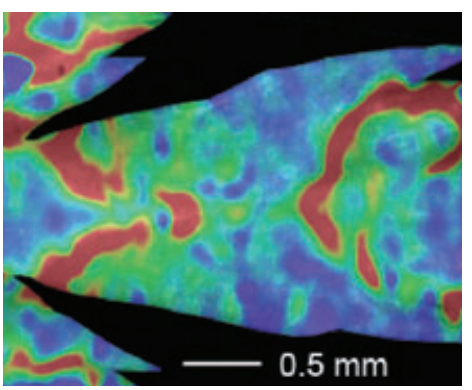

\section{C. formosensis}
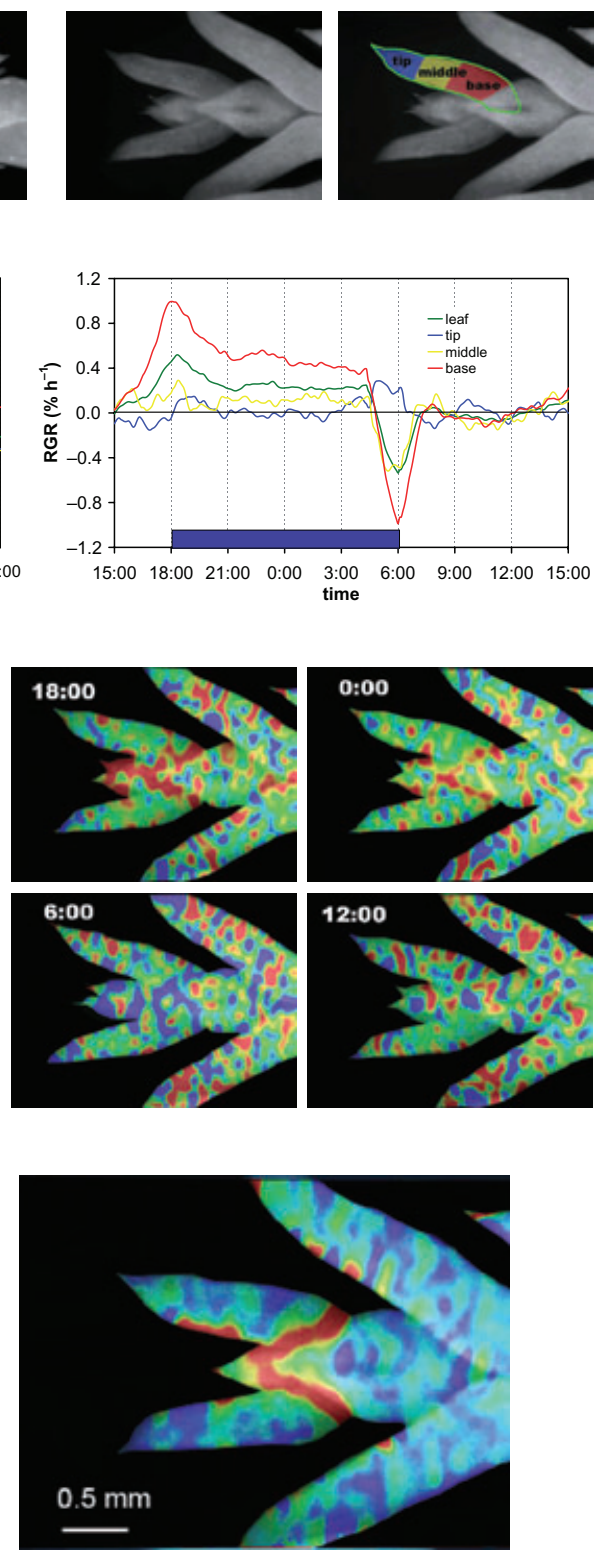

Figure 4. Spatial change of diurnal growth rate (RGR) from one typical leaf of

Chamaecyparis obtusa var. formosana and $C$. formosensis under medium light regime. (a) First original image of the sequence. Insets show areas of interest that were chosen to analyse growth at the tip (blue), middle (yellow), and base (red) region of the leaf. Green line: area of interest depicting an entire leaf. (b) Time series of the diurnal growth cycle in selected leaf regions. (c) Colourcoded distributions of relative growth rate at four points in time. Blue (low) to red (high) colours show a range of $\pm 2.5 \% \mathrm{~h}^{-1}$ in C. obtusa var. formosana and $\pm 1.8 \% \mathrm{~h}^{-1}$ in $C$.

formosensis. (d) Colour-coded distribution of the daily average of relative growth rate within the analysed foliage. Blue (low) to red (high) colours show a range of -0.2 to $1.2 \% \mathrm{~h}^{-1}$ in C. obtusa var. formosana and -0.2 to $0.6 \% \mathrm{~h}^{-1}$ in C. formosensis. throughout the morning hours (0600-1100 h). In contrast to the behaviour of $C$. obtusa var. formosana, the diurnal growth rate patterns of $C$. formosensis showed an almost identical shape for all three light regimes.

Differences in general growth behaviour were most apparent when RGR was averaged throughout the entire day ( $n=30$ or more, Fig. 7). Chamaecyparis obtusa var. formosana grew at a higher rate than $C$. formosensis in the low light regime $(P<0.001)$, whereas $C$. formosensis showed higher growth rates than $C$. obtusa var. formosana in the medium light regime $(P<0.001)$ and also in the high light regime, although the difference was not significant $(P=0.288)$. Comparison of the average growth rates between light intensities showed that growth rates in the medium light intensity were at least a factor of two higher than in low or high light conditions for both species. For
C. obtusa var. formosana, mean growth rates did not differ between low and high light intensity, whereas C. formosensis showed significantly higher growth rates in the high light regime compared to the low light regime $(P<0.001)$.

\section{Transfer of plants between light regimes}

Foliage growth was followed throughout $12 \mathrm{~d}$ in this experiment with plants growing for the first $6 \mathrm{~d}$ in conditions to which they were acclimated and for the last $6 \mathrm{~d}$ in altered light conditions (Fig. 8; $n=2$ ). This experiment revealed another difference between the diurnal growth behaviour of the two species: C. obtusa var. formosana showed normal, co-ordinated growth rhythms in both light conditions with a much lower amplitude in low light. When light conditions were switched, the amplitude of the plant formerly 


\section{C. obtusa var. formosana}

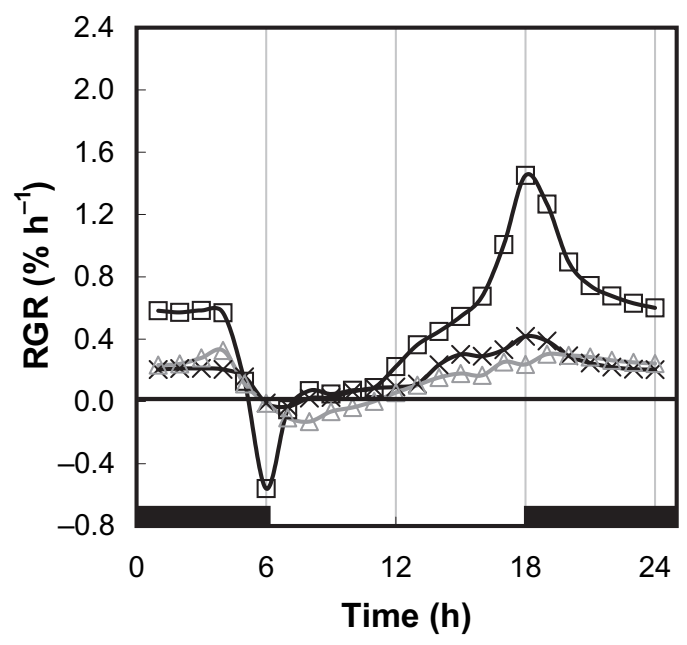

C. formosensis

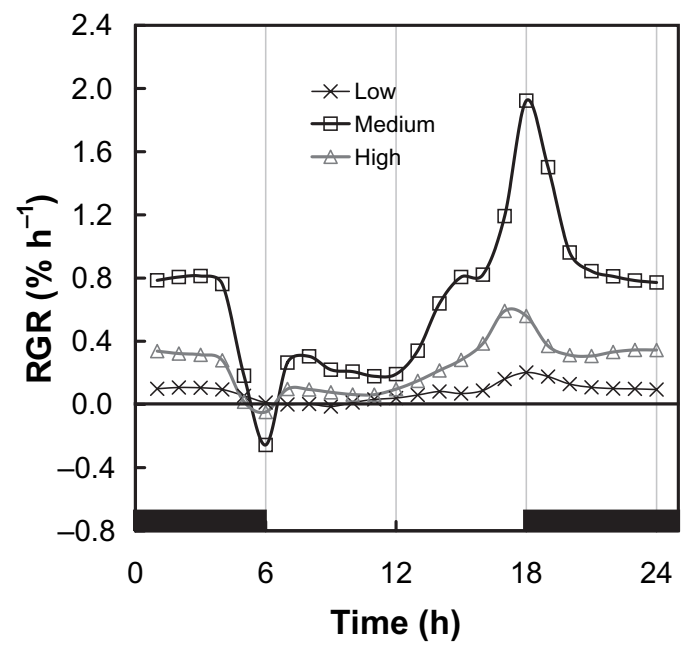

Figure 5. Time course of relative growth rate (RGR) of the entire leaf under different light regimes for Chamaecyparis obtusa var. formosana and $C$. formosensis $(n=38,43,31$ for C. obtusa var. formosana in low, medium, and high light, respectively, and $n=40$, 48, and 58 for C. formosensis, respectively).

in medium light conditions decreased within $1 \mathrm{~d}$, but the growth rhythm remained. The plants that were transferred to medium light conditions increased their growth amplitude within $24 \mathrm{~h}$.

Chamaecyparis formosensis leaves grown in low light conditions showed very small amplitudes of diurnal growth fluctuations, and medium light conditions resulted in a normal diurnal cycle. Upon transfer of the plants to the other condition, the plants from medium light conditions showed fading growth intensity throughout the entire $6 \mathrm{~d}$, reaching values comparable to those that were reached by C. obtusa var. formosana at day 2 , only at day 6 . The plants that were switched to medium light conditions showed continuously increasing growth intensity and also needed $6 \mathrm{~d}$ to reach comparable growth cycles as C. obtusa var. formosana at day 2 after switching.

\section{DISCUSSION}

The results of this study show that the investigated congener species differ in leaf growth dynamics and that those differences in their growth kinetics reflect the most important features of their natural light environment. The species adapted to grow under closed canopies (C. obtusa var. formosana) is much more dynamic (Fig. 8) and grows more
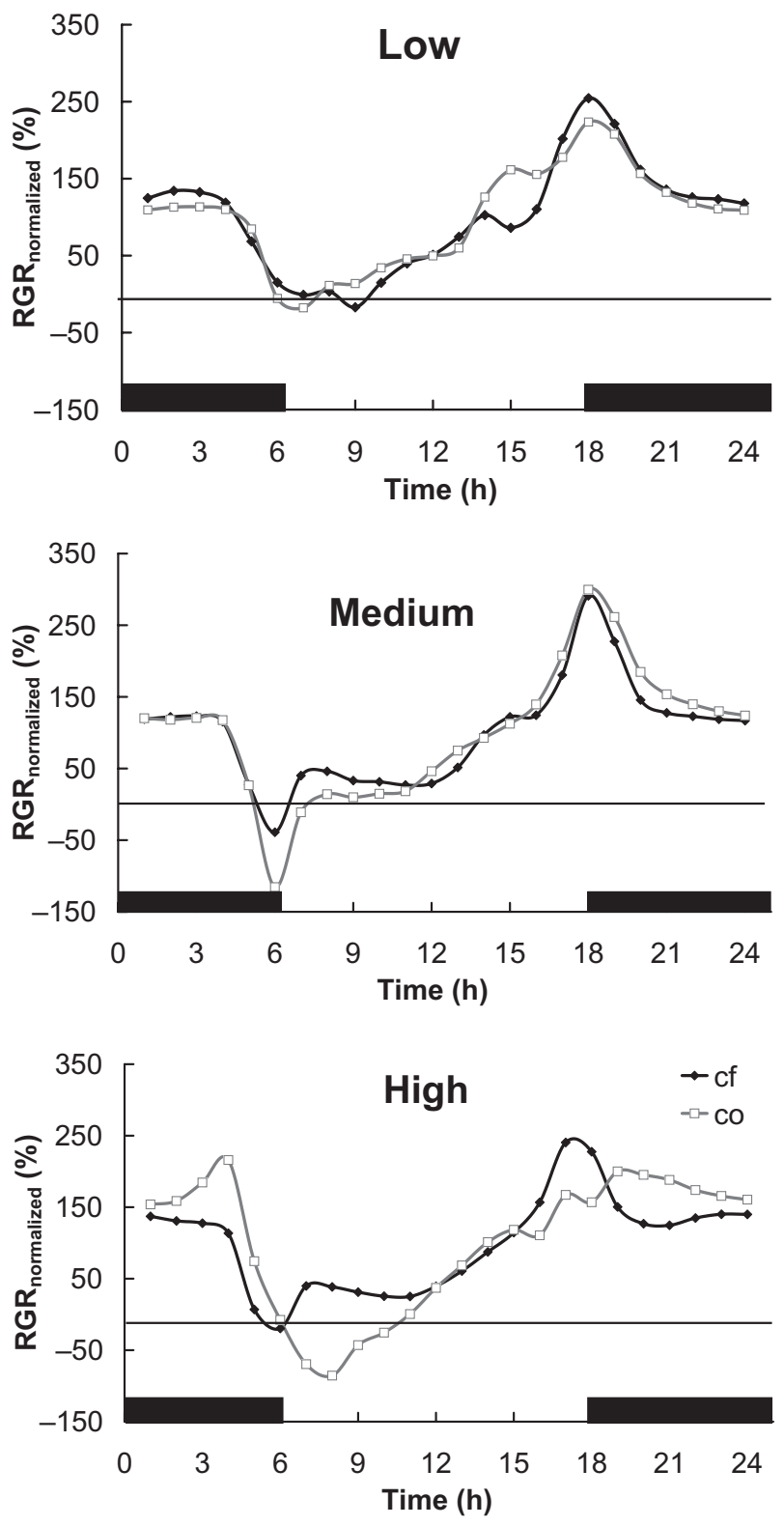

Figure 6. Normalized relative growth rate (RGR) of

Chamaecyparis obtusa var. formosana (co) and C. formosensis (cf) under different light regimes. Values are calculated from the data shown in Fig. 5 by normalizing RGR-values for each replicate to the average value throughout $24 \mathrm{~h}$ (average $\mathrm{RGR}=100 \%$ ). 


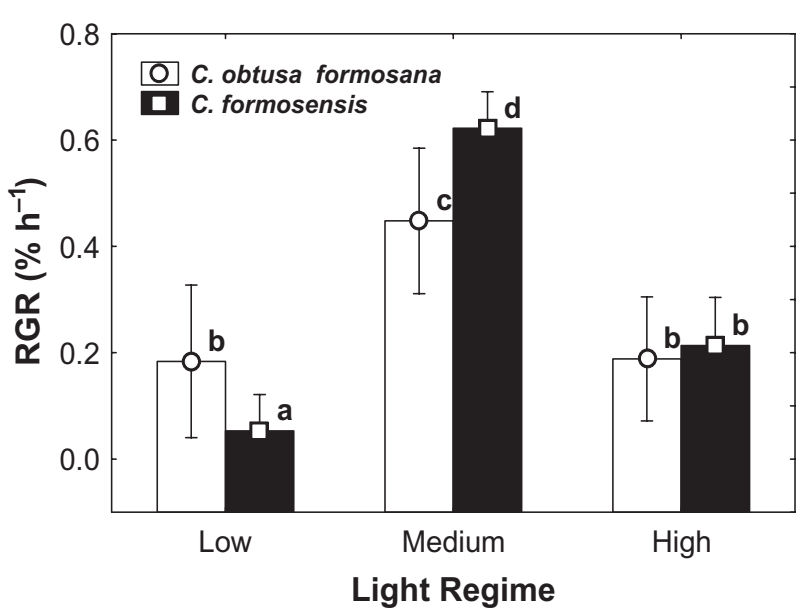

Figure 7. Comparison of the average relative growth rate (RGR) of Chamaecyparis obtusa var. formosana and C. formosensis under different light regimes. The data were calculated from the daily average RGR of all replicates that are included in the data of Fig. 5 (standard deviation and results of Fisher's LSD test are shown).

successful in low light conditions (Figs 5 \& 7). In constantly high light, its leaves shrink throughout an extended period of the day (Figs $5 \& 6$ ). Hence, this species seems to be adapted to highly fluctuating but overall shady conditions, as is the case in the understorey of a forest. In contrast to this, the species adapted to open vegetation situations,
C. formosensis, has lower growth rates in low light conditions (Figs $5 \& 7$ ), and reacts much slower towards changes of light intensity (Fig. 8). Chamaecyparis formosensis seems to tolerate higher light conditions better than C. obtusa var. formosana, as can be concluded from the finding that it does not show negative growth rates under high light conditions. The sudden onset of artificial light in the morning will surely cause changes in leaf water relations, such as stomatal conductance, water potential, and turgor. The kinetics with which those plant traits can recover from suddenly imposed, stressful conditions might be closely related to the kinetics of sudden leaf growth responses and hence to the overall growth performance of a plant. Growth rates of $C$. formosensis were also higher in the medium light regime in comparison to C. obtusa var. formosana. Hence, C. formosensis is better adapted to an open vegetation situation with overall moderate light intensities, which corresponds to the distribution of $C$. formosensis seedlings in their natural site.

Photosynthetic strategies also adapt $C$. formosensis to the moderate light intensities in cloud forests (Fig. 2). $A_{\max }$, $R_{\mathrm{d}}$ and $Q_{\mathrm{y}}$ are inhibited in the high light conditions applied in this study; leaves reach their optimal physiological state at moderate PPFD-conditions. The fact that - in the medium light regime $-C$. formosensis has a higher $A_{\max }$ compared with $C$. obtusa var. formosana might indicate that $C$. formosensis can build up more reserves from photosynthesis, enabling a slower decay of growth rate after
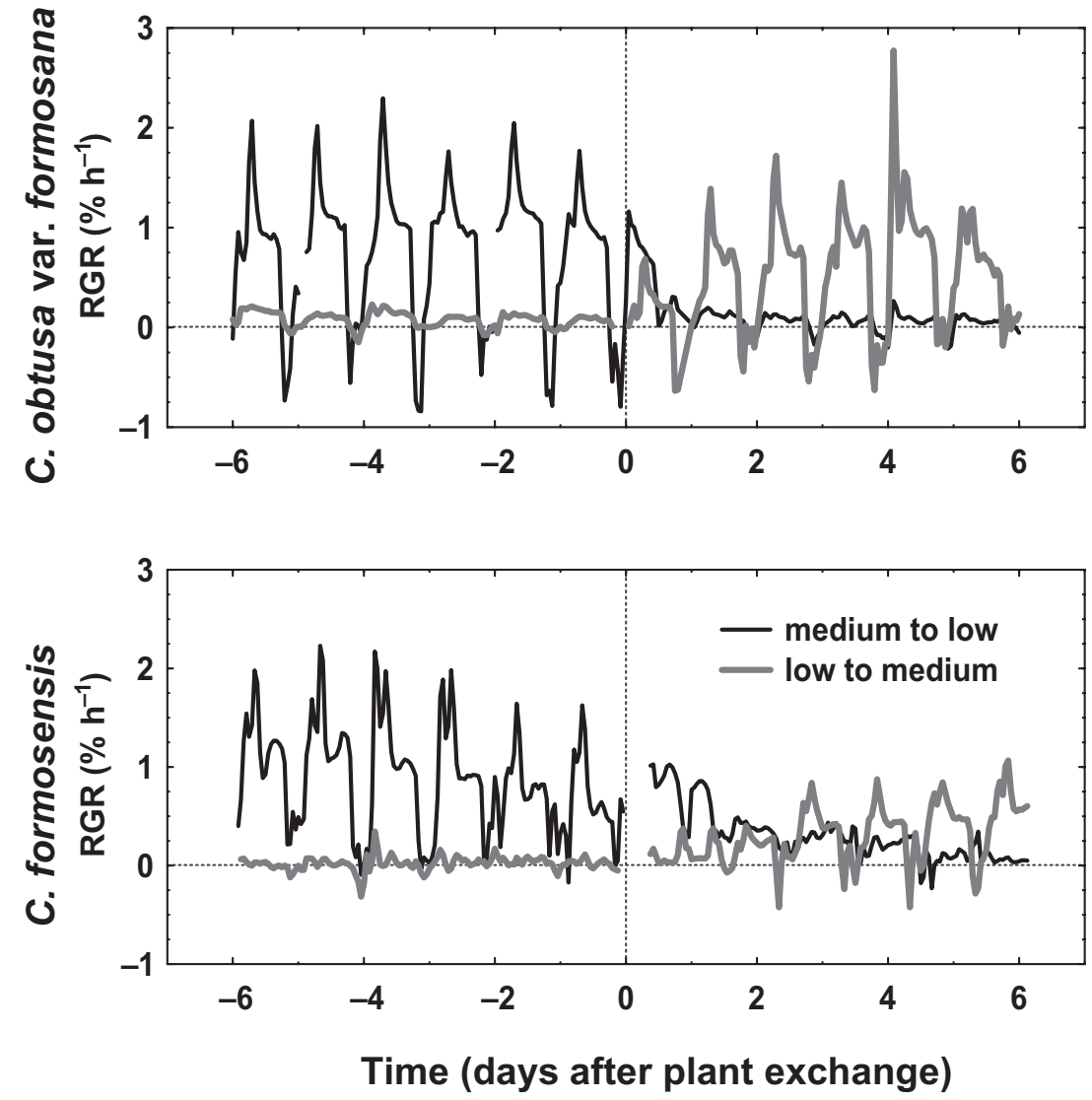

Figure 8. Time series of relative growth rate (RGR) during light change experiments for Chamaecyparis obtusa var. formosana and $C$. formosensis $(n=2)$. 
transition to a lower light regime. Although the applied light regimes with a constant illumination throughout $12 \mathrm{~h}$ were different from natural light conditions, they mimicked average light intensities at typical growing sites and the results confirmed findings from nursery management. There, it is well known that planted seedlings need a generally shady environment (Lin \& Lin 2000). Vegetation surveys show clearly that seedlings of C. formosensis are predominantly found in open gaps or severely disturbed areas (Chang 1963; Liu 1975; Lin \& Lai 1999). There, the prevailing fog will typically lead to moderate PPFD and so the optimal growth conditions for this species will most probably be on foggy and not on clear days as has been expected previously.

In summary, the present findings indicate that a detailed analysis of leaf growth dynamics can be a useful tool to verify the ecological amplitude of a given species in much the same way as this can be done via measurements of photosynthetic acclimation of the investigated species (Murchie \& Horton 1998). However, causal explanations for the ecological amplitude of a given species cannot be expected from analysis of a single trait alone, since the capacity to adjust to a given situation is always connected to a wide range of traits. Moreover, it has to be noted that the results obtained here for two congener gymnosperm species do not allow general conclusions for other species. Yet, those results might be a first step towards the extraction of 'growth acclimation strategies' that are similar to photosynthetic acclimation strategies that were elucidated for a wider range of angiosperms (Murchie \& Horton 1997).

The basic rhythm of diurnal growth activity does not differ between the two species investigated here, indicating that the same mechanisms govern the growth dynamics in both species. However, the diurnal rhythmicity reported here shows antiphasic behaviour in comparison with the results from leaf growth in Ricinus communis (Walter, Feil \& Schurr 2002a) or Nicotiana tabacum (Walter \& Schurr 2005) that show maximal growth intensity in the late night. This supports the view that a network of mechanisms is responsible for the regulation of growth intensity throughout $24 \mathrm{~h}$, with different weights of certain mechanisms in different species.

Biophysically, growth is regulated by the interaction between internal plant pressure (turgor) as the driving force and the rigidity (or extensibility) of the cell wall as the retarding force (Lockhart 1965; Cosgrove 1986). The controlling mechanisms have to alter these biophysical properties in a diurnally regulated manner. Carbohydrate metabolism is one of those mechanisms affecting diurnal growth patterns (Kehr et al. 1998; Walter \& Schurr 2005). Other mechanisms include changes in the activity of cellwall loosening factors such as expansin and xyloglucanendo-transglycosylase (Cosgrove 1999). Moreover, sudden changes in light intensity do exert fast effects on, for example, cell wall pH (Mühling et al. 1995) and thus may affect cell wall extensibility. They also affect stomatal conductance (Mott \& Buckley 2000) and may thus influence tur- gor. Yet, the connection between all these elements of the network regulating diurnal growth variations is still unclear. This study shows that comparison of growth patterns between congener species occupying different ecological niches is another means of elucidating connections within the regulatory network as differences in growth dynamics are connected to differences in physiology of these species. In future studies, it will be important to clarify at the level of metabolites, enzymes and gene activities, why shade-tolerant species can make faster use of their growth potential in dynamically changing light conditions, why they are shrinking in the early morning hours at high light and why shade-avoiding species adapted to open vegetation situations can use more of their growth potential in constant situations of moderate PPFD.

The differences observed in growth dynamics of the two species correlate with the ecological amplitude in light intensity that each species experiences under natural conditions. This leads to the conclusion that a detailed analysis of growth dynamics is a helpful tool for investigating physiological differences between species and that it can also serve as a helpful tool in predicting plant competitiveness under given environmental situations by clarifying the variance of the ecological amplitude of a given species.

\section{ACKNOWLEDGEMENTS}

We are grateful for the financial support by a grant to IL.L. from the international co-operation 'Sandwich Program' for Ph.D. candidates by German Academic Exchange Service (DAAD) and the National Science Council of Taiwan (NSC). We acknowledge provision of seedlings by the 'Forest Protection Department under the Veterans Affairs Commission of the Executive Yuan of the Republic of China'. We thank Lisa Ainsworth and Shizue Matsubara for helpful comments on the manuscript, Michael Schilbach and Beate Uhlig for help with plant cultivation, and Maja Christ for assistance with the imaging software.

\section{REFERENCES}

Anderson J.M., Park Y.I. \& Chow W.S. (1997) Photoinactivation and photoprotection of photosystem II in nature. Physiologia Plantarum 100, 214-223.

Bailey S., Walters R.G., Jansson S. \& Horton P. (2001) Acclimation of Arabidopsis thaliana to the light environment: the existence of separate low light and high light responses. Planta 213, 794-801.

Barker M. \& Brown N. (1997) Photosynthetic characteristics of dipterocarp seedlings in three tropical rain forest light environments: a basis for niche partitioning? Oecologia 112, 453463.

Barth C., Krause C.G. \& Winter K. (2001) Responses of phytosystem I compared with photosystem II to high-light stress in tropical shade and sun leaves. Plant, Cell and Environment 24, 163-176.

Bassow S.L. \& Bazzaz F.A. (1997) Intra- and inter-specific variation in canopy photosynthesis in a mixed deciduous forest. Oecologia 109, 507-515. 
Chang L.M. (1963) Ecological Studies of Chamaecyparis formosensis and Chamaecyparis taiwanensis mixed forest in Taiwan. The Bulletin of Taiwan Forestry. Research Institute no. 91. Taiwan Forestry Research Institute, Taipei, Taiwan. (in Chinese with English abstract).

Chen C.D. (1968) The selection of planted tree species in Taiwan. Quarterly Journal of Chinese Forestry 1, 79-86. (in Chinese).

Cosgrove D.J. (1986) Biophysical control of plant cell growth. Annual Review of Plant Physiology 37, 377-405.

Cosgrove D.J. (1999) Enzymes and other agents that enhance cell wall extensibility. Annual Review of Plant Physiology and Plant Molecular Biology 50, 391-417.

Dalling J.W., Lovelock C.E. \& Hubbell S.P. (1999) Growth responses of two neotropical pioneer seedlings to simulated forest gap environments. Journal of Tropical Ecology 15, 827-839.

Davies S.L. (1998) Photosynthesis of nine pioneer Macaranga species from Borneo in relation to life history. Ecology 79, 2292 2308.

Demmig-Adams B. (1998) Survey of thermal energy dissipation and pigment composition in sun and shade leaves. Plant Cell Physiology 39, 474-482.

Demmig-Adams B. \& Adams W.W.I.I.I. (1992) Photoprotection and other responses of plants to high light stress. Annual Review of Plant Physiology and Plant Molecular Biology 43, 599-626.

Ho C.F. (1988) The inbreeding of Chamaecyparis formosensis forest. Modern Silviculture 3, 24-28. (in Chinese).

Kehr J., Hustiak F., Walz C., Willmitzer L. \& Fisahn J. (1998) Transgenic plants changed in carbon allocation pattern display a shift in diurnal growth pattern. Plant Journal 16, 497-503.

Lee S.C. (1962) Taiwan red-and yellow-cypress and their conservation. Taiwania. 8, 1-13.

Lei T.T. \& Lechowicz M.J. (1990) Shade adaptation and shade tolerance in saplings of three Acer species from eastern North America. Oecologia 84, 224-228.

Lei T.T. \& Lechowicz M.J. (1998) Diverse responses of maple saplings to forest light regimes. Annals of Botany 82, 9-19.

Lin C.H. \& Lai K.S. (1999) The structure and composition of Chamaecyparis formosensis forest in Juiyen River. Endemic Species Research 1, 43-60. (in Chinese with English abstract).

Lin Y.J. \& Lin J.L. (2000) The Breeding of Chamaecyparis seedlings. Taiwan Forestry Research Institute, Taipei, Taiwan. (in Chinese).

Liu T. (1975) The study of community ecology of Chamaecyparis formosensis and Chamaecyparis taiwanensis mixed forest in Taiwan. Journal of Agricultural Association of China 92, 143-178.

Lockhart J.A. (1965) An analysis of irreversible plant cell elongation. Journal of Theoretical Biology 8, 264-275.

Mott K.A. \& Buckley T.N. (2000) Patchy stomatal conductance: emergent collective behaviour of stomata. Trends in Plant Science 5, 258-262.

Mühling K.H., Plieth C., Hansen U.P. \& Sattelmacher B. (1995) Apoplastic $\mathrm{pH}$ of intact leaves of Vicia faba as influenced by light. Journal of Experimental Botany 46, 377-382.

Murchie E.H. \& Horton P. (1997) Acclimation of photosynthesis to irradiance and spectral quality in British plant species: Chlorophyll content, photosynthetic capacity and habitat preference. Plant, Cell and Environment 20, 438-448.

Murchie E.H. \& Horton P. (1998) Contrasting patterns of photosynthetic acclimation to the light environment are dependent on the differential expression of the responses to altered irradiance and spectral quality. Plant, Cell and Environment 21, $139-148$.

Niinemets U., Bilger W., Kull O. \& Tenhunen J.D. (1998) Acclimation to high irradiance in temperate deciduous trees in the field: Changes in xanthophyll cycle pool size and in photosynthetic capacity along a canopy light gradient. Plant, Cell and Environment 21, 1205-1218.

Oguchi R., Hikosaka K. \& Hirose T. (2003) Does the photosynthetic light-acclimation need change in leaf anatomy? Plant, Cell and Environment 26, 505-512.

Öquist G., Anderson J.M., McCaffery S. \& Chow W.S. (1992) Mechanistic differences in photoinhibition of sun and shade plants. Planta 188, 422-431.

Scharr H. (2004) Optimal filters for extended optical flow. International Workshop on Complex Motion. Springer, Heidelberg, Günzburg, Germany.

Schmundt D., Stitt M., Jähne B. \& Schurr U. (1998) Quantitative analysis of the local rates of growth of dicot leaves at a high temporal and spatial resolution, using image sequence analysis. Plant Journal 16, 505-514.

Su H.J. (1984) Studies on the climate and vegetation types of the natural forests in Taiwan. II. Altitudinal vegetation zones in relation to temperature gradient. Quarterly Journal of Chinese Forestry 17, 57-73.

Walter A., Feil R. \& Schurr U. (2002a) Restriction of nyctinastic movements and application of tensile forces to leaves affects diurnal patterns of expansion growth. Functional Plant Biology 29, 1247-1258.

Walter A. \& Schurr U. (2005) Dynamics of leaf and root growth endogenous control versus environmental impact. Annals of Botany 95, 891-900.

Walter A., Spies H., Terjung S., Küsters R., Kirchgeßner N. \& Schurr U. (2002b) Spatio-temporal dynamics of expansion growth in roots: automatic quantification of diurnal course and temperature response by digital image sequence processing. Journal of Experimental Botany 53, 689-698.

Walters R.G. (2005) Towards an understanding of photosynthetic acclimation. Journal of Experimental Botany 56, 435-447.

van der Weele C.M., Jiang H.S., Palaniappan K.K., Ivanov V.B., Palaniappan K. \& Baskin T.I. (2003) A new algorithm for computational image analysis of deformable motion at high spatial and temporal resolution applied to root growth. Roughly uniform elongation in the meristem and also, after an abrupt acceleration, in the elongation zone. Plant Physiology 132,11381148.

Received 8 March 2005; received in revised form 9 May 2005; accepted for publication 11 May 2005 